\title{
Dielectrically enhanced excitons in semiconductor-insulator quantum wires: Theory and experiment
}

\author{
E. A. Muljarov \\ General Physics Institute, Russian Academy of Sciences, Vavilova Street 38, Moscow 117942, Russia \\ E. A. Zhukov \\ M. V. Lomonosov Moscow State University, 119899 Moscow, Russia \\ and Single Quantum Dot Project, ERATO, JST, Tsukuba Research Consortium, Tsukuba, Ibaraki 300-2635, Japan \\ V. S. Dneprovskii \\ M. V. Lomonosov Moscow State University, 119899 Moscow, Russia \\ Yasuaki Masumoto \\ Single Quantum Dot Project, ERATO, JST, Tsukuba Research Consortium, Tsukuba, Ibaraki 300-2635, Japan \\ and University of Tsukuba, Tsukuba, Ibaraki 305-8571, Japan
}

(Received 10 September 1999)

\begin{abstract}
We present both theoretical and experimental investigations of optical properties of excitons in semiconductor-insulator quantum wires. Spectra of linear and nonlinear absorption, photoluminescence and its polarization, photoluminescence excitation, time-resolved photoluminescence of GaAs, CdSe, and InP quantum wires 4-6 nm in diameter, crystallized in dielectric matrix, demonstrate the prominent excitonic behavior. In these structures an essential difference of dielectric constants of constituent materials leads to a considerable enhancement of excitons, the binding energies ranging from $120 \mathrm{meV}$ to $260 \mathrm{meV}$ and exciton transitions being well distinguished in nanowires with sufficient dispersion of diameter even at room temperature. A theoretical approach to calculations of the exciton parameters in a semiconductor-insulator cylindrical quantum wire of finite diameter is developed. This approach accounts for a band-gap renormalization due to the spatial confinement and self-image effect, as well as for a dielectric enhancement of the electron-hole interaction. The calculated exciton transition energies and absorption spectra are consistent with the experimental results.
\end{abstract}

\section{INTRODUCTION}

The investigation of low-dimensional semiconductor structures, such as quantum wires (QWR's), has attracted much attention in recent years, ${ }^{1-5}$ because they not only demonstrate interesting electronic and excitonic properties, but also show promise for their application to electronic and optoelectronic devices. ${ }^{1,2}$ In QWR's, carriers and excitons move freely in one direction but are confined in two others. This results in prominent exciton features such as large binding energies $\left(E_{\mathrm{b}}\right)$ and oscillator strengths: the restriction of motion reduces the distance between an electron and a hole, thus enhancing their effective Coulomb attraction. It is well known that the binding energy of a particle in a onedimensional (1D) Coulomb potential is infinite. In real QWR's the exciton binding energy can be evaluated as ${ }^{6} E_{\mathrm{b}}$ $\propto \mathrm{Ry}^{*}\left[\ln \left(R / a_{\mathrm{B}}^{*}\right)\right]^{2}$, where $R$ is a wire radius and $a_{\mathrm{B}}^{*}$ and $\mathrm{Ry} *$ are the effective atomic units in a bulk. Thus, even in rather thin quantum wires the two-dimensional confinement itself leads to an increase of the binding energy only a few times $^{3-5,7,8}$ (up to $20-30 \mathrm{meV}$ for typical semiconductor QWR's). These values, however, can be significantly increased if a semiconducting barrier material of a QWR structure is replaced by an insulator with a smaller dielectric constant. ${ }^{9,10}$ In such a system the electric field induced by carriers becomes significantly redistributed due to a dielectric constant mismatch, so that the contribution of the insu- lator to the electron-hole interaction enlarges the exciton binding energy.

The effect of the dielectric enhancement of excitons, also referred to in literature as dielectric confinement, is a wellknown phenomenon. It was theoretically predicted in the original papers ${ }^{11-13}$ that excitonic properties of semiconductor thin films surrounded by a dielectric or vacuum should be much more pronounced than that in a bulk. Later this phenomenon was theoretically investigated in quantum wells, ${ }^{14-19}$ superlattices, ${ }^{20,21}$ QWR's, ${ }^{8-10}$ and quantum dots. ${ }^{22}$ Until recently the study of the dielectric enhancement of excitons in real semiconductor nanostructures was restricted to the case when the dielectric constant mismatch is small (of the order of 10\%), and the effect is rather weak. ${ }^{16-18}$ Even in GaAs/pure AlAs T-shaped QWR's, the maximum enhancement of the exciton binding energy due to the dielectric constant mismatch ranges up to $25 \%$, as it was recently shown in Ref. 8. In semiconductor-insulator nanostructures this effect is clearly demonstrated by measurements of large exciton binding energies and oscillator strengths. ${ }^{23,24}$ Varying the difference of dielectric constants of constituent parts of a nanostructure it is possible to change the binding energy and oscillator strengths of excitons, thus realizing Coulomb interaction engineering. ${ }^{6}$ In order to increase the Coulomb interaction it is also reasonable to place a semiconductor structure near the interface with vacuum, as it has been realized, for example, in open quantum wires ${ }^{25}$ 
and near-surface quantum wells, ${ }^{26,27}$ or to add a neighboring dielectric layer. ${ }^{28}$ However, in such structures it is not possible to increase the electron-hole interaction too much, since a sufficient dielectric surrounding cannot be prepared by technical reasons. In semiconductor quantum wires embedded into an insulator matrix the effect of the dielectric enhancement of excitons becomes the most prominent for at least two reasons. First, in nanostructures with QWR's it is possible to build up a sizable dielectric surrounding. Second, excitons are sufficiently extended (unlike to quantum dots) along the free motion axis, providing that the largest share of the electromagnetic field is brought into dielectric.

In the present paper we investigate excitonic properties of $\mathrm{GaAs}, \mathrm{CdSe}$, and InP quantum wires crystallized in a transparent dielectric matrix: chrysotile asbestos nanotubes. ${ }^{29,30}$ We report a study of linear and nonlinear absorption, photoluminescence (PL), photoluminescence excitation (PLE), time-resolved PL, and polarization properties of the PL. We show that in these nanostructures the role of the dielectric enhancement is of paramount importance that leads to large values of the exciton binding energy ranging from $120 \mathrm{meV}$ to $260 \mathrm{meV}$ in these materials. Large oscillator strengths of exciton transitions make it possible to observe the latter even at room temperature and under conditions of a considerable inhomogeneous broadening. Due to a large difference (a factor of 3 to 5) between the dielectric constants of semiconductor and the matrix, image potentials play an essential role in the exciton binding and determines together with the dimensional quantization the quasi-one-dimensional character of excitons. These potentials lead to the effect of the dielectric enhancement (the exciton binding energy increases by a factor of several tens compared to that in a bulk) and to a renormalization of QWR-localizing potentials owing to additional self-image terms. ${ }^{15,16,21}$ A theoretical approach developed in this paper allows us to calculate excitonic parameters in a semiconductor-insulator cylindrical quantum wire of finite diameter, unlike the previous approaches, ${ }^{9,10,24}$ which have been devoted to extremely thin QWR's. The band-gap remormalization, exciton binding energies, and absorption spectra are calculated with accounting for the full image-charge-magnified electrostatic potential of an electron-hole system.

The paper is organized as follows. In Sec. II the details of sample preparation and characterization as well as of optical measurements are given. Section III is devoted to a theoretical model of a Wannier exciton in a semiconductorinsulator cylindrical QWR. In Sec. IV the experimental results are given and discussed in relation to the theoretical calculations.

\section{EXPERIMENTAL DETAILS}

There are various techniques for fabrication of QWR's, namely, the molecular-beam epitaxy (MBE) or metal-organic chemical vapor-phase deposition (MOCVD) on preprocessed substrates, etching of two-dimensional semiconductor structures, and cleaving of a two-dimensional structure in a plane perpendicular to the surface with continued MBE process on the cleaved surface (fabrication of a T-shaped QWR), etc. These techniques, however, do not allow someone to produce samples in which the dimensions and density of QWR's are suitable to measure linear absorption spectra without an optical near-field microscope. We have investigated the samples with semiconductor nanostructures that were manufactured using the following methods: (i) melted GaAs was injected in hollow nanometer channels of chrysotile asbestos tubes and then crystallized; ${ }^{30}$ (ii) CdSe was synthesized in the nanochannels of chrysotile asbestos using water solution of $\mathrm{Cd}(\mathrm{COOH})_{2}$ and $\mathrm{H}_{2} \mathrm{Se}$ according to the chemical reaction $\mathrm{Cd}(\mathrm{COOH})_{2}+\mathrm{H}_{2} \mathrm{Se}=\mathrm{CdSe}+2 \mathrm{HCOOH} ;{ }^{31}$ (iii) InP nanostructures were crystallized in chrysotile asbestos using the MOCVD technique. ${ }^{32}$ Parameters of nanotubes were measured by a high-resolution electron transmission microscope (for more details see in Refs. 30 and 31). The samples represent bundles of densely packed regular structures of parallel chrysotile asbestos nanotubes up to several millimeters long with external diameters of about $30 \mathrm{~nm}$ and internal diameters of 4-6nm, which are arranged hexagonally and stacked together by amorphous silica. The samples in which $\mathrm{GaAs}$ and CdSe wires were grown contain nanotubes of different types: most of them have an inside diameter of $4.8 \mathrm{~nm}$, the rest have diameters of about $4 \mathrm{~nm}$ and $6 \mathrm{~nm}$. The samples with InP nanostructures have inside diameters of $4-4.8 \mathrm{~nm}$. The deposition of semiconducting material was mainly formed in the channels of nanotubes. As it is shown in Ref. 30 , in such nanotubes most of semiconductor $(\mathrm{GaAs})$ is crystallized in the form of wires with an average length of about $30 \mathrm{~nm}$ and the rest is crystallized as much shorter quantumdotlike nanostructures. A minor amount of semiconductor is crystallized between the bundles of nanotubes. ${ }^{33}$

The PL spectra were measured at a sample temperature of 2 and $300 \mathrm{~K}$ using Ar-ion laser $(2.54 \mathrm{eV}, 1 \mathrm{~mW})$ or $\mathrm{He}-\mathrm{Cd}$ laser $(3.81 \mathrm{eV}, 5 \mathrm{~mW})$ for excitation, $0.275 \mathrm{~m}$ polichromator (Spectra Pro-275, Acton Research Corporation) with a 300 $\mathrm{g} / \mathrm{mm}$ diffraction grating and a charge-coupled device camera (LN/CCD - 1100, Princeton Instruments Inc.) cooled by liquid nitrogen. The laser beam was focused on a spot with a diameter of about $200 \mu \mathrm{m}$.

The time-resolved spectra of PL were registered using a Ritsu MC-12N polychromator and Hamamatsu C1587 synchroscan streak camera with a two-dimensional detector. The focused beam of the second harmonic of the Argon-ion pumped Ti-sapphire laser $(\hbar \omega=3.1 \mathrm{eV}$, a pulse duration of $1.5 \mathrm{ps}$, a repetition rate of $82 \mathrm{MHz}$, and the energy density of $0.2 \mu \mathrm{J} / \mathrm{cm}^{2}$ ) was used for excitation of nanostructures. The time resolution was better than $50 \mathrm{ps}$ and the spectral resolution was about $1 \mathrm{meV}$.

The excitation spectrum was obtained using a Xe lamp and two Jasco CT-25CP monochromators with $600 \mathrm{~g} / \mathrm{mm}$ gratings. One of the monochromators was used for selection and tuning the wavelength of the exciting beam. The other was utilized together with photon counting system for registration of a PLE signal at $\hbar \omega=1.909 \mathrm{eV}$. The spectral resolution of the system was $5 \mathrm{meV}$.

In our experiments we used the pump and probe method for measurements of the differential transmission

$$
D T(\omega)=\frac{T(\omega)-T_{0}(\omega)}{T_{0}(\omega)},
$$

where $T(\omega)$ and $T_{0}(\omega)$ are the transmission spectra of the excited and unexcited sample. 
The GaAs and CdSe quantum wires were excited by ultrashort optical pulses (with a duration of about $20 \mathrm{ps)} \mathrm{of} \mathrm{the}$ second harmonic of a Nd:YAG (yttrium aluminum garnet) mode-locked laser $(\hbar \omega=2.33 \mathrm{eV})$. The pump intensity was up to $100 \mathrm{MW} / \mathrm{cm}^{2}$. The pumping-laser beam polarized in the direction parallel to the quantum wires was normal to the sample surface and focused into a spot $200 \mu \mathrm{m}$ in diameter. The central part of the excited spot was probed by the focused polarized "white" light. In order to generate the ultrashort white probing pulse, a part of the laser beam (at the fundamental frequency) was fed to a cell filled with heavy water. An optical delay line allowed us to delay the probing pulse from the pumping-laser pulse and study the kinetics of changes induced by the pumping radiation. The spectrum of the probing pulse ("picosecond continuum") was recorded both in front of and behind the sample using an OVA-284 optical multichannel analyzer.

\section{THEORY}

Our theoretical approach is based on the model ${ }^{10}$ of Wannier-Mott exciton localized in a cylindrical semiconductor wire inside a dielectric matrix. Due to a large band gap of the insulator (approximately $4 \mathrm{eV}$ in chrysotile asbestos) and additional self-image potentials, which grow up rapidly in a semiconductor near the interface, we can consider carriers as being strongly localized in a two-dimensional potential of the quantum wire. Thus, we use the ansatz

$$
\boldsymbol{\Psi}\left(\boldsymbol{\rho}_{e}, \boldsymbol{\rho}_{h}, z\right)=\mathcal{R}_{e}\left(\rho_{e}\right) \mathcal{R}_{h}\left(\rho_{h}\right) \psi(z),
$$

where $z$ is the electron-hole relative coordinate along the wire, $\boldsymbol{\rho}_{e, h}$ is a radius vector of an electron (hole) in the $x y$ plane, and $\mathcal{R}_{e, h}(\rho)$ is the normalized wave function of the ground state of a QWR-localized electron (hole). In the framework of the effective-mass approximation, which accounts for a spatially dependent (stepwise) dielectric constant, ${ }^{16,21}$ the zero-momentum exciton Hamiltonian takes the form

$$
\begin{aligned}
\hat{H}= & -\frac{\hbar^{2}}{2}\left(\frac{1}{m_{e}^{\perp}} \nabla_{\boldsymbol{\rho}_{e}}^{2}+\frac{1}{m_{h}^{\perp}} \nabla_{\boldsymbol{\rho}_{h}}^{2}+\frac{1}{\mu} \frac{\partial^{2}}{\partial z^{2}}\right) \\
& +U_{e}\left(\rho_{e}\right)+U_{h}\left(\rho_{h}\right)+V_{e h}\left(\boldsymbol{\rho}_{e}, \boldsymbol{\rho}_{h}, z\right),
\end{aligned}
$$

where $U_{e, h}$ is the electron (hole) localizing potential including both conduction (valence) band offset and self-image interaction, $V_{e h}$ is the image-potential electron-hole interaction, $m_{e}^{\perp, \|}, m_{h}^{\perp, \|}$, and $\mu$ are, respectively, effective masses of an electron and a hole and a reduced excitonic mass $1 / \mu$ $=1 / m_{e}^{\|}+1 / m_{h}^{\|}$. The signs $\|$and $\perp$ refer, respectively, to quantities along and normal to the wire axis $z$.

We assume the effective masses to be anisotropic due to a strong-confinement regime, when the valence band formerly degenerate in a bulk semiconductor is split off and the effects of band nonparabolicity are suppressed. In this situation the effective-mass anisotropy of heavy and light holes can be approximately treated $\mathrm{as}^{34}$

$$
\begin{array}{ll}
m_{h h}^{\perp}=m_{0}\left(\gamma_{1}-2 \gamma_{2}\right)^{-1}, & m_{h h}^{\|}=m_{0}\left(\gamma_{1}+\gamma_{2}\right)^{-1}, \\
m_{l h}^{\perp}=m_{0}\left(\gamma_{1}+2 \gamma_{2}\right)^{-1}, & m_{l h}^{\|}=m_{0}\left(\gamma_{1}-\gamma_{2}\right)^{-1},
\end{array}
$$

where $m_{h h}^{\perp, \|}$ and $m_{l h}^{\perp, \|}$ are, respectively, heavy and light hole masses, $m_{0}$ is the free-electron mass, and $\gamma_{1}, \gamma_{2}$ are the Luttinger parameters. ${ }^{35}$ Thus, within our theoretical model we consider two types of excitons: an $h h$ exciton, formed by an electron and a hole that is light along the wire axis $z$ and heavy in the $x y$ plane where its motion is confined, and an $l h$ exciton, which is formed with participation of a hole that is heavy along the wire axis and light in the confinement plane.

\section{A. Self-image potentials and QWR-confined states}

To calculate one-particle ground-state wave functions $\mathcal{R}_{e, h}(\rho)$ and the renormalized semiconductor band gap, we solve for each type of particle the following Schrödinger equation:

$$
\begin{aligned}
& {\left[-\frac{\hbar^{2}}{2 m_{e, h}^{\perp}}\left(\frac{\partial^{2}}{\partial \rho^{2}}+\frac{1}{\rho} \frac{\partial}{\partial \rho}\right)+U_{e, h}^{0}(\rho)+U_{\mathrm{self}}(\rho)\right] \mathcal{R}_{e, h}(\rho)} \\
& \quad=E_{e, h} \mathcal{R}_{e, h}(\rho),
\end{aligned}
$$

where $U_{e, h}^{0}$ is the heterostructure potential for the electron (hole) and $U_{\text {self }}$ is the self-image potential. The latter is the same for both particles and takes the form

$$
\begin{aligned}
U_{\text {self }}(\rho) & =\frac{e}{2} \lim _{\boldsymbol{\rho}^{\prime} \rightarrow \boldsymbol{\rho}}\left[\varphi\left(\rho, \rho^{\prime}, \theta, 0\right)-\frac{e}{\varepsilon_{i} \sqrt{\left(\boldsymbol{\rho}-\boldsymbol{\rho}^{\prime}\right)^{2}}}\right] \\
& =\frac{e}{2} \int_{-\infty}^{\infty} d k \sum_{n=-\infty}^{\infty} \Phi_{n}^{\text {hom }}(\rho, \rho, k),
\end{aligned}
$$

where potentials $\varphi\left(\rho, \rho^{\prime}, \theta, z\right)$ and $\Phi_{n}^{\text {hom }}\left(\rho, \rho^{\prime}, k\right)$ are given by Eqs. (A3)-(A10) (see Appendix A), $\varepsilon_{i}=\varepsilon_{\mathrm{S}}$ inside the wire $\rho \leqslant R$, and $\varepsilon_{i}=\varepsilon_{\mathrm{I}}$ outside the wire $\rho \geqslant R$. Here $\varepsilon_{\mathrm{S}, \mathrm{I}}$ is the semiconductor (insulator) dielectric constant, and $R$ is the wire radius.

As soon as $\rho$ approaches $R, U_{\text {self }}(\rho)$ goes to infinity on both sides of the semiconductor-insulator interface. Since we assume the dielectric constant changes abruptly at the interface, the Coulomb-like divergencies occur when a charge encounters its image. In a semiconductor the charge is repulsed from the interface and in a dielectric it is attracted to the interface. To avoid this unphysical behavior we cut off the self-image potential at a height of the heterostructure potential in the barrier, as shown in Fig. 1 (thick solid lines). These drastic changes, however, occur at the distances of several angstroms and do not affect the quantum state in a wide QWR. As the barrier is rather high in a typical semiconductor-insulator QWR we do not account for the self-image potential in the insulator area. However, in rather thin QWR's it should be taken into account as the electron (hole) states become more sensitive to the local changes in potential. In this case the divergencies may be avoided by introducing a transition layer in which the potential is defined finite. In thin wires the image-potential divergencies 


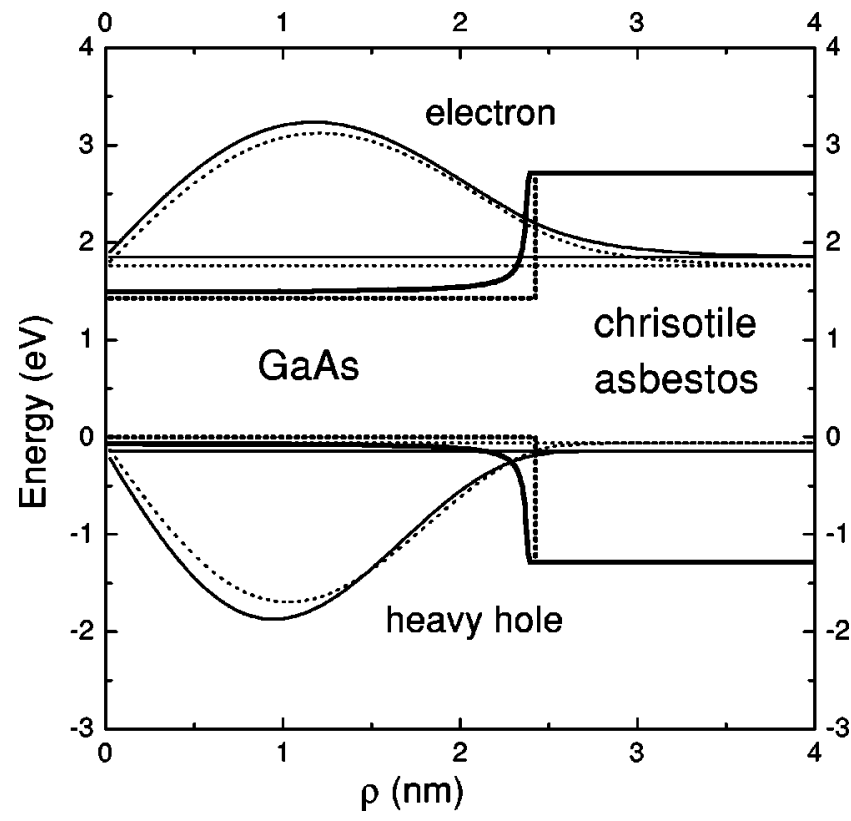

FIG. 1. QWR potentials (thick lines), electron and hole probabilities $\rho \mathcal{R}_{e, h}^{2}(\rho)$, and energy levels (thin lines) of the dimensional quantization with and without self-image potentials (solid and dashed curves, respectively), calculated for $R=2.4 \mathrm{~nm}$ GaAs QWR in chrysotile asbestos.

could even result in the formation of a new type of states that are located at the interface due to additional minima in the barrier. $^{21}$

The self-image potentials essentially modify the quantum well shape by reducing its depth and growing up its minimum (compare with the heterostructure image-free potentials also shown in Fig. 1 by dotted lines). This affects primarily the semiconductor band-gap renormalization, hence the exciton peak position. Being almost unimportant in rather wide QWR's, the effect of band-gap renormalization becomes more and more crucial as $R$ becomes smaller: in GaAs QWR's in chrysotile asbestos with $R=2.4 \mathrm{~nm}$ it is as large as $175 \mathrm{meV}$. The repulsion of charges from the interface leads to not only growing up the minimum of the well and, as a consequence, a blueshift (tens to hundreds of meV) of the band gap, but also to significant changes of the radial wave functions that in turn affects the exciton binding energy.

The two-dimensional potentials simulated for the investigated QWR's are deep enough to localize two or more oneparticle states for electrons and holes. The separation between dimensional quantization levels varies from 100$150 \mathrm{meV}$ for heavy holes to several hundreds of $\mathrm{meV}$ for light holes and electrons. These values are comparable to or larger than the calculated exciton binding energies and much larger than the Coulomb correlation energy between dimensional quantization levels. This justifies our choice of the wave function Eq. (2) with separated variables.

\section{B. Variational approach}

In rather thin quantum wires the exciton binding energy is well described within a simple variational approach. ${ }^{10}$ Let us choose the wave function of the exciton relative motion as

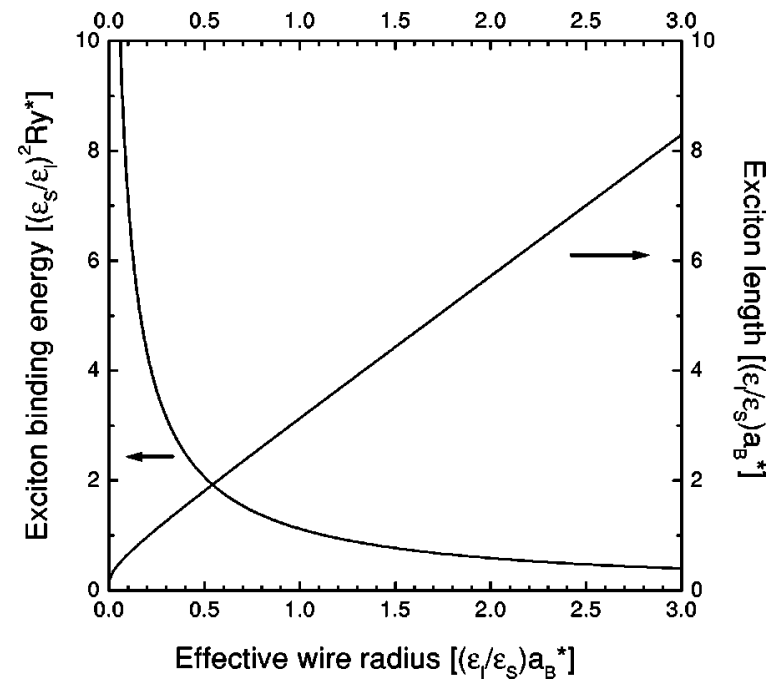

FIG. 2. Calculated variationally exciton binding energy and averaged length vs effective wire radius $R_{\text {eff }}$ (for details see the text). Insulator atomic units $\left(\varepsilon_{\mathrm{S}} / \varepsilon_{\mathrm{I}}\right)^{2} \mathrm{Ry} \mathrm{y}^{*}$ and $\left(\varepsilon_{\mathrm{I}} / \varepsilon_{\mathrm{S}}\right) a_{\mathrm{B}}^{*}$ are used, where $\mathrm{Ry}^{*}=\mu e^{2} / 2 \hbar^{2} \varepsilon_{\mathrm{S}}^{2}$ and $a_{\mathrm{B}}^{*}=\hbar^{2} \varepsilon_{\mathrm{S}} / \mu e^{2}$ are semiconductor atomic units.

$$
\psi(z)=\frac{a^{1 / 2}}{\pi^{1 / 4}} \exp \left(-\frac{1}{2} a^{2} z^{2}\right),
$$

where $a$ is the variational parameter, and assume that the wire radius is much smaller than the mean exciton length, namely,

$$
\frac{\varepsilon_{\mathrm{S}}}{\varepsilon_{\mathrm{I}}}(a R)^{2}|\ln (a R)| \ll 1 .
$$

This means that the cylindrical functions involved in the Fourier transform of the electrostatic potential [Eqs. (A6)(A10) (see Appendix A)] can be replaced by their asymptotic values at small $k$, thus resulting in the approximation Eq. (A11). Thereafter the integrats over $z$ and $k$ in the Hamiltonian expectation value are calculated analytically and the functional of the exciton energy (measured from the energy of unbound electron-hole pair in the QWR) takes the form

$$
E(a)=\frac{\hbar^{2}}{4 \mu} a^{2}+\frac{2 e^{2}}{\varepsilon_{\mathrm{I}}} \frac{a}{\sqrt{\pi}}\left(\ln \frac{a R_{\mathrm{eff}}}{2}+\frac{1}{2} C\right),
$$

where $C$ is Euler's constant and $R_{\text {eff }}$ is the effective wire radius defined as

$$
\begin{aligned}
R_{\mathrm{eff}} & =\operatorname{Rexp}\left\{\frac{\varepsilon_{\mathrm{I}}}{\varepsilon_{\mathrm{S}}} S(R)+S(\infty)-S(R)\right\}, \\
S(x)= & (2 \pi)^{2} \int_{0}^{x} \rho d \rho \ln \frac{\rho}{R} \int_{0}^{\rho} \rho^{\prime} d \rho^{\prime} \\
& \times\left\{\mathcal{R}_{e}^{2}(\rho) \mathcal{R}_{h}^{2}\left(\rho^{\prime}\right)+\mathcal{R}_{e}^{2}\left(\rho^{\prime}\right) \mathcal{R}_{h}^{2}(\rho)\right\} .
\end{aligned}
$$

In Fig. 2 the exciton binding energy and mean length are shown as functions of $R_{\text {eff }}$ and measured in the effective atomic units renormalized to the barrier dielectric constant, i.e., in $\left(\varepsilon_{\mathrm{S}} / \varepsilon_{\mathrm{I}}\right)^{2} \mathrm{Ry}^{*}$ and $\left(\varepsilon_{\mathrm{I}} / \varepsilon_{\mathrm{S}}\right) a_{\mathrm{B}}^{*}$. Note that in these units 
the excitonic parameters calculated through a minimization of $E(a)$ can be expressed by universal dependencies (shown in Fig. 2), the structural parameters being concentrated in $R_{\text {eff }}$. The effective wire radius $R_{\text {eff }}$ also depends on the spatial localization of carries in the cross-section plane of the wire. However, due to almost logarithmic behavior ${ }^{10}$ of $\min \{E(a)\}$ this dependence is rather weak.

As it is clear from Eq. (9) the exciton binding energy is determined by the insulator dielectric constant, since the main part of the electric field is concentrated in the insulator. This is just the case of a rather elongated exciton that is formed in thin QWR's.

\section{Effective image-potential electron-hole interaction}

In thick wires the variational approach does not give satisfactory results as $\Phi_{n}\left(\rho, \rho^{\prime}, k\right)$ contributes to the electronhole interaction at large $k$. Thus, the full electrostatic potential has to be taken into account. The exciton dimension along the axis of free motion is of the order of $R$ and a fraction of the Coulomb field concentrated in semiconductor becomes more and more perceptible. However, the twodimensional quantum wells for electrons and holes are still deep enough to justify the ansatz Eq. (2).

The image-potential mediated electron-hole interaction can be written as

$$
\begin{aligned}
V(z)= & \iint V_{e h}\left(\boldsymbol{\rho}, \boldsymbol{\rho}^{\prime}, z\right) \mathcal{R}_{e}^{2}(\rho) \mathcal{R}_{h}^{2}\left(\rho^{\prime}\right) d \boldsymbol{\rho} d \boldsymbol{\rho}^{\prime} \\
= & -e \int_{-\infty}^{\infty} d k e^{i k z}(2 \pi)^{2} \int_{0}^{\infty} \int_{0}^{\infty} \Phi_{0}\left(\rho, \rho^{\prime}, k\right) \\
& \times \mathcal{R}_{e}^{2}(\rho) \mathcal{R}_{h}^{2}\left(\rho^{\prime}\right) \rho d \rho \rho^{\prime} d \rho^{\prime},
\end{aligned}
$$

where we have used (as in Sec. III B) the fact that $\mathcal{R}_{e, h}^{2}(\rho)$ is an axially symmetric function. The exciton wave function $\psi(z)$ obeys the Schrödinger equation

$$
\left[-\frac{\hbar^{2}}{2 \mu} \frac{d^{2}}{d z^{2}}+V(z)-E\right] \psi(z)=0,
$$

which determines the electron-hole relative motion.

The ground-state solution of Eq. (13), namely, the exciton binding energy and mean length versus $R$, is shown in Fig. 3 (solid lines) for different values of dielectric constant ratio. The enhancement of the exciton binding energy due to the dielectric constant mismatch is visible (note that the curves with $\varepsilon_{\mathrm{I}} / \varepsilon_{\mathrm{S}}=1$ correspond to the case when the effect of image charges is absent). Unlike the variational solution, it is not possible to bring these dependencies to any universal form since the full image potential is now taken into account. For comparison, the excitonic parameters obtained in our variational approach (Sec. III B) are shown by dotted lines. For small $R$ the variational energies and lengths satisfactory fit the numerical solution, though it is obvious that for $\varepsilon_{\mathrm{I}} / \varepsilon_{\mathrm{S}}=1$ (no images) the binding energy calculated variationally is smaller, and for $\varepsilon_{\mathrm{I}} / \varepsilon_{\mathrm{S}}=0.1$ (strong dielectric confinement) it is overestimated since only a part of the full image potential is included into Eq. (9) [see also Eq. (A11)]. Another variational solution for the exciton binding energy taken from Refs. 6 and 9 for $\varepsilon_{\mathrm{I}} / \varepsilon_{\mathrm{S}}=0.1$ is also shown in Fig.
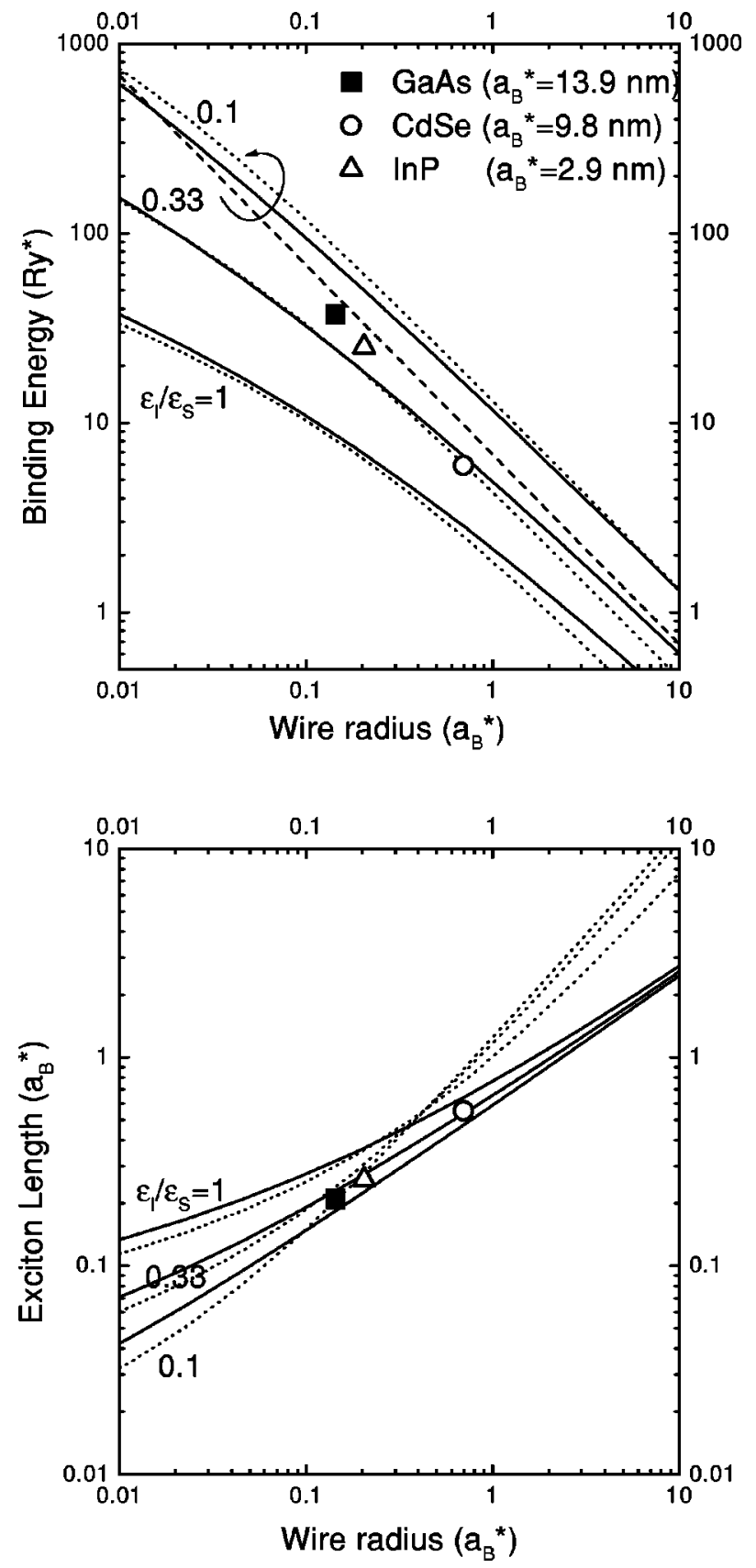

FIG. 3. Exciton binding energies (top) and exciton mean length (bottom) as a function of the wire radius $R$ calculated with the account for the full image potential for different values of the dielectric constant ratio (solid curves). The same for $\mathrm{GaAs}, \mathrm{CdSe}$, and InP QWR $4 \mathrm{~nm}$ in diameter (solid squares, open circles and triangles). The same in the limit of a thin QWR within the variational approaches of Ref. 10 (dotted curves) and Refs. 6 and 9 (dashed curve).

3 by a dashed line. However, the variational approach of Refs. 6 and 9 is found to be much more crude than that used in the present paper.

Using the numerical solution of Eq. (13) we have calculated the energies of excitonic transitions in $\mathrm{InP}, \mathrm{GaAs}$, and CdSe QWR's with the diameter of $4 \mathrm{~nm}, 4.8 \mathrm{~nm}$, and $6 \mathrm{~nm}$ for further comparison in Sec. IV with experimental spectra of linear absorption, differential transmission, and photoluminescence. The exciton binding energies and lengths calcu- 
lated for these types of QWR's that are $4 \mathrm{~nm}$ in diameter are also plotted in Fig. 3.

In our calculations we use the following bulk semiconductor parameters. ${ }^{36}$ For GaAs QWR's: the electron effective mass $m_{e}=0.067 m_{0}$, the Luttinger parameters $\gamma_{1}=6.95, \gamma_{2}$ $=2.25$, direct energy gap $E_{g}=1.428 \mathrm{eV}(T=300 \mathrm{~K})$, and the high frequency dielectric constant ${ }^{37} \varepsilon_{\mathrm{S}}=10.9$; for InP QWR's: $m_{e}=0.079 m_{0}, \gamma_{1}=4.94, \quad \gamma_{2}=1.65, E_{g}=1.4236$ $\mathrm{eV}(T=2 \mathrm{~K})$, and $\varepsilon_{\mathrm{S}}=9.61$; for CdSe QWR's: $m_{e}$ $=0.12 m_{0}$, the hole effective masses and energy gap of, respectively, sub-bands $A$ and $B$ are $(T=300 \mathrm{~K}) m_{\perp}^{A}$ $=0.45 m_{0}, m_{\|}^{A}=1.0 m_{0}, E_{g}^{A}=1.751 \mathrm{eV}$, and $m_{\perp}^{B}=0.9 m_{0}$, $m_{\|}^{B}=1.0 m_{0}, E_{g}^{B}=1.771 \mathrm{eV}, \varepsilon_{\mathrm{S}}=5.8$. Following Ref. 38 we assume for chrysotile asbestos $E_{g}=4 \mathrm{eV}$ and $\varepsilon_{\mathrm{I}}=2.2$. We would like to note that no adjustable parameters have been used in the calculations, except that we assume the effectivemass parameters of the dielectric being the same as in semiconductor.

\section{Exciton absorption spectrum}

One of the most intrinsic properties of semiconductorinsulator nanostructures, which manifests itself in the optical spectra, is a significant anisotropy of the optical signal with respect to the polarization of the applied electromagnetic field. 25,39

Let us consider a QWR subjected to an electromagnetic field with the wavelength $\lambda$ much larger than the wire radius. Within the dipole approximation the absorption and luminescence are proportional to the scalar product of the local electric field $\mathbf{E}$ and interband dipole momentum $\mathbf{d}$, averaged over the full electron-hole wave function. In semiconductorinsulator nanostructures, $\mathbf{E}$ strongly depends on the orientation of the electric field $\mathbf{E}_{\infty}$ far from the nanostructure. Taking into account that $2 R \ll \lambda$, we can treat the light electromagnetic field inside the sample as a plane wave with an amplitude modulated on the scale of the wire radius, due to the usual boundary conditions. When $\mathbf{E}_{\infty}$ is parallel to the wire, no such modulation takes place. However, the local field $\mathbf{E}$ is strongly modulated when $\mathbf{E}_{\infty}$ is normal to the wire axis. Inside the wire the electric field components have the form $^{40}$

$$
\mathbf{E}^{\|}=\mathbf{E}_{\infty}^{\|}, \quad \mathbf{E}^{\perp}=\delta \mathbf{E}_{\infty}^{\perp},
$$

where $1-\delta$ is a depolarization factor, and

$$
\delta=\frac{2 \varepsilon_{\mathrm{I}}}{\varepsilon_{\mathrm{I}}+\varepsilon_{\mathrm{S}}} .
$$

The absorption coefficient $\alpha(\omega)$ is proportional to $\langle\mathbf{E} \cdot \mathbf{d}\rangle^{2} / E_{\infty}^{2}$. Taking into account that the light is emitted and absorbed in semiconductor only, we immediately find that the linear polarization degree of the absorption has the form

$$
\sigma=\frac{\alpha^{\|}-\alpha^{\perp}}{\alpha^{\|}+\alpha^{\perp}}=\frac{\left|\mathbf{d}_{c v}^{\|}\right|^{2}-\delta^{2}\left|\mathbf{d}_{c v}^{\perp}\right|^{2}}{\left|\mathbf{d}_{c v}^{\|}\right|^{2}+\delta^{2}\left|\mathbf{d}_{c v}^{\perp}\right|^{2}} .
$$

If we assume that the dipole matrix element $\mathbf{d}_{c v}$ between the conduction and valence bands is isotropic, then

$$
\sigma=\frac{1-\delta^{2}}{1+\delta^{2}}
$$

Finally, in the case of a very large difference between the dielectric constants $\left(\varepsilon_{\mathrm{I}} / \varepsilon_{\mathrm{S}} \ll 1\right)$ we obtain the maximum anisotropy,

$$
\sigma \approx 1-8 \frac{\varepsilon_{\mathrm{I}}}{\varepsilon_{\mathrm{S}}} .
$$

This effect, of course, is only slightly manifested in quantum dots whose form is more or less spherical and vanishes in purely spherical objects where the field redistribution due to boundary conditions does not depend on the exciting light polarization.

We have considered the polarization anisotropy which contributes to the exciton absorption spectrum. Let us now find the optical susceptibility $\chi(\omega)$ which does not depend on the magnitude of the electromagnetic field. We are interested in the contribution of both bound and scattering states to the exciton absorption spectrum. As soon as we know the magnified electron-hole potential $V(z)$, Eq. (12), it is reasonable to introduce the exciton Green's function $G\left(z, z^{\prime}, E\right)$, which obeys an inhomogeneous Schrödinger equation [Eq. (13)] with a $\delta$ function in the right-hand side. Then,

$$
\chi(\omega)=-f_{0} \lim _{z \rightarrow 0} G\left(z, z ; \hbar \omega-E_{g}+i \Gamma\right)
$$

with

$$
f_{0}=2 \pi\left|\mathbf{d}_{c v}\right|^{2}\left|\int_{0}^{R} \mathcal{R}_{e}(\rho) \mathcal{R}_{h}(\rho) \rho d \rho\right|^{2},
$$

where $E_{g}$ is the QWR-renormalized band gap (calculated in Sec. III A), and the broadening $\Gamma$ is taken into account. $G(0,0, E)$ can be expressed ${ }^{41}$ in terms of a solution $y(z ; E)$ of the homogeneous equation [Eq. (13)] as

$$
G(0,0 ; E)=\frac{-y(0 ; E)}{y^{\prime}(0 ; E)},
$$

where $E=\hbar \omega-E_{g}+i \Gamma, y(z ; E)$ decays exponentially at large distances. In our approach the function $y(z ; E)$ is a numerical solution of Eq. (13) treating the complex energy $E$ as a parameter. The results of these calculations are discussed in Secs. IV B and IV D (see below).

\section{EXPERIMENTAL RESULTS AND DISCUSSIONS}

\section{A. Exciton transitions in absorption, differential transmission, and photoluminescence spectra}

Spectra of linear absorption of GaAs, CdSe, and InP QWR's grown inside chrysotile asbestos nanotubes are given, respectively, in Figs. 4, 5, and 6, curves $a$. Note the features in the linear absorption spectrum which are clearly distinguished on a monotonously growing background of the absorption, namely, broad bands peaking at around the frequencies of excitation transitions calculated within the theoretical model and shown by solid (for $h h$ excitons in GaAs and InP and $A$-excitons in CdSe) and dashed arrows (for $l h$ 


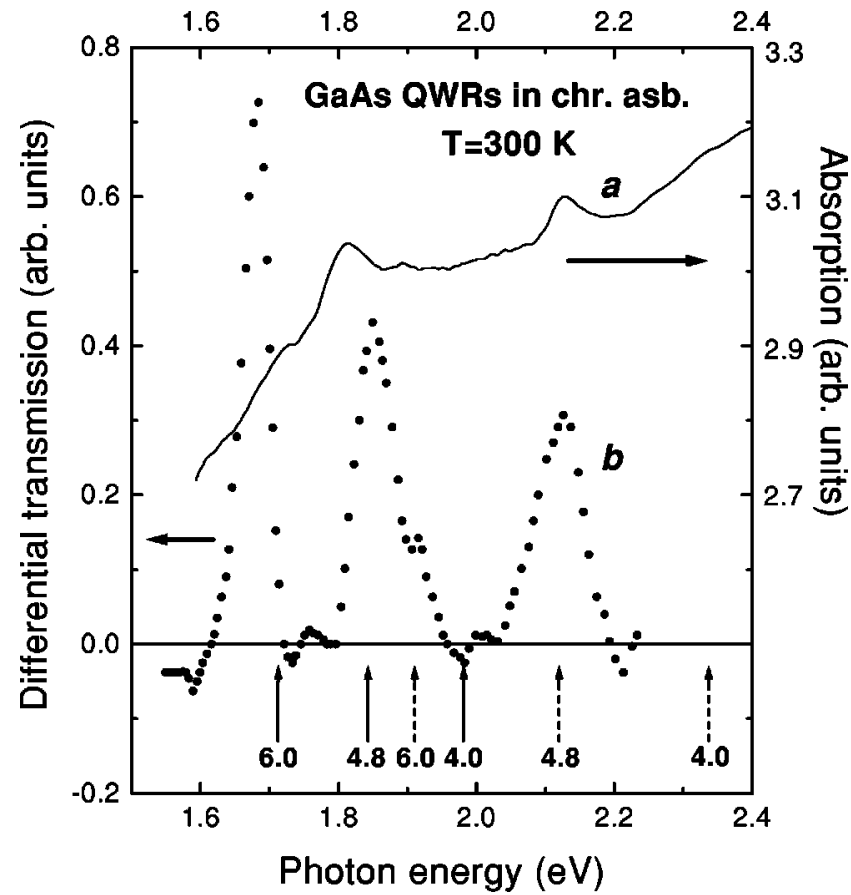

FIG. 4. Spectra of linear absorption (curve $a$ ) and differential transmission (curve $b$ ) at zero delay between the pumping and probing pulses of GaAs in chrysotile-asbestos nanotubes. The solid and dashed arrows show the calculated exciton transitions for $h h$ and $l h$ excitons, respectively, in QWR's with diameters of $4 \mathrm{~nm}$, $4.8 \mathrm{~nm}$, and $6 \mathrm{~nm}$.

and $B$ excitons). In the absorption spectrum of GaAs nanostructures (Fig. 4, curve $a$ ) the observed broad bands have maxima at $1.82 \mathrm{eV}, 1.89 \mathrm{eV}$, and $2.11 \mathrm{eV}$ as well as a "shoulder" in the vicinity of $1.7 \mathrm{eV}$. The spectrum of the sample containing CdSe (Fig. 5, curve $a$ ) has two broad bands: a high-energy band at $1.95-2.05 \mathrm{eV}$ and a low-energy band at around $1.8 \mathrm{eV}$. A broad band centered at $1.92 \mathrm{eV}$ is observed in the linear absorption spectra of chrysotile asbestos samples containing InP (Fig. 6, curve $a$ ).

The differential transmission (DT) spectrum of GaAs QWR's measured at zero delay between the pumping and probing pulses (Fig. 4, curve $b$ ) contains bleaching bands at $1.67 \mathrm{eV}, 1.85 \mathrm{eV}$ and $2.14 \mathrm{eV}$. The positions of induced bleaching bands in the DT spectrum coincide with the corresponding hills in the linear absorption spectrum. The lowenergy band (at $1.67 \mathrm{eV}$ ) in the DT spectra of GaAs QWR's has been registered only for some parts of the samples (the exciting and probing beams were scanned across the sample surfaces). The DT spectra of CdSe QWR's registered at different delay between the pump and probe pulses (Fig. 5, curves $b$ and $c$ ) contain two main bleaching bands: a shortwave band peaking at $2.05 \mathrm{eV}$ and a long-wave band at $1.79 \mathrm{eV}$.

The photoluminescence (PL) spectra of InP nanostructures crystallized in chrysotile asbestos nanotubes contain three bands centered at $2.295 \mathrm{eV}, 1.892 \mathrm{eV}$, (Fig. 6, curves $b$ and $c$ ) and $1.796 \mathrm{eV}$. The positions of these bands almost do not depend on temperature. The third band that is absent in two typical spectra shown in Fig. 6, was detected only in some regions of the samples. It may be explained by electron-hole transitions in InP quantum-dotlike structures (see discussion below). The high-energy band at $2.295 \mathrm{eV}$

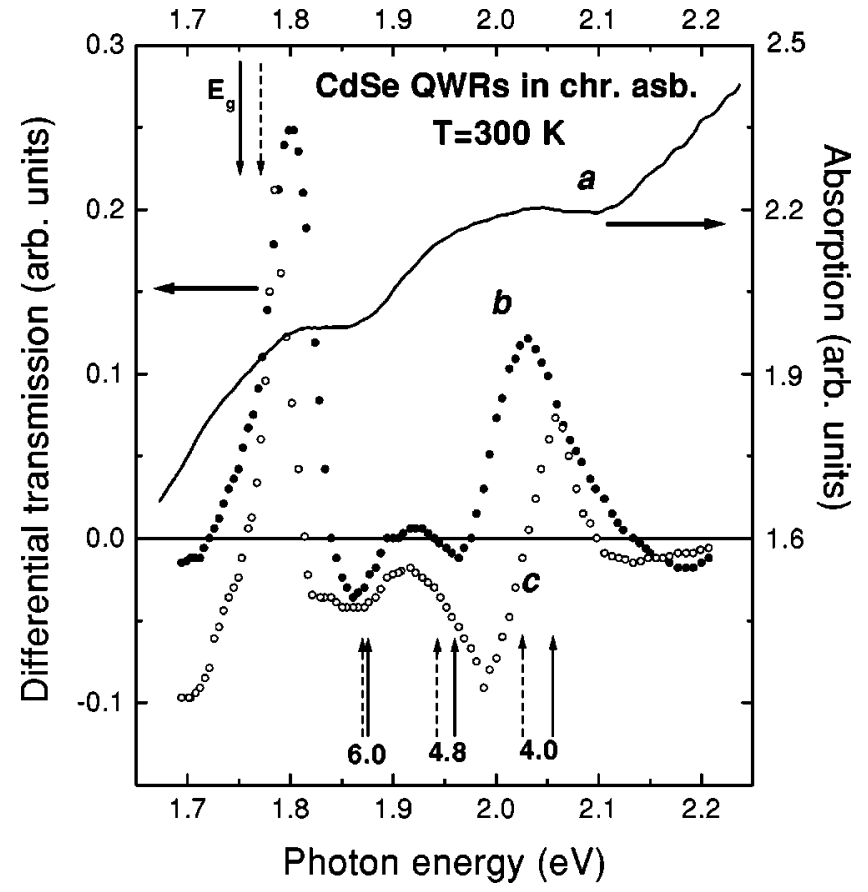

FIG. 5. Spectra of linear absorption (curve $a$ ) and differential transmission at zero delay (curve $b$ ) and 7 ps delay (curve $c$ ) between the pumping and probing pulses of CdSe in chrysotileasbestos nanotubes. The solid and dashed up arrows show the calculated exciton transitions for $A$ and $B$ excitons formed with the participation of $A$ and $B$ hole sub-bands, respectively, in QWR's with diameters of $4 \mathrm{~nm}, 4.8 \mathrm{~nm}$, and $6 \mathrm{~nm}$. The solid and dashed down arrows show the band-gap energies of $A$ and $B$ sub-bands of bulk CdSe.

probably belongs to relaxation of electron excited states trapped on defects in the insulator ${ }^{33}$ (oxygen vacancies) that emit far below the energy gap of chrysotile asbestos [compare with PL spectrum of pure chrysotile asbestos with empty nanochannels, (Fig. 6, curve $d$ )]. Finally, the middle band centered at $1.892 \mathrm{eV}$ is close to the corresponding band in the absorption spectrum, Fig. 6, curve $a$, and most probably originates from the excitonic transitions in QWR's. Unfortunately, we are not able to estimate the Stokes shift of the PL from the absorption peak because of a strong inhomogeneous broadening of the exciton absorption band. The excitation and PL detected from a small area (about $2 \mu \mathrm{m}$ in diameter) allows us to exclude partly the inhomogeneous broadening of the exciton PL band, while the cooling of the samples from $300 \mathrm{~K}$ to $2 \mathrm{~K}$ leads to minor changes in PL.

Unlike three- and two-dimensional semiconductor systems, optical properties of quasi-one-dimensional systems are largely controlled by excitonic transitions owing to an anomalously large concentration of oscillator strengths at frequencies of excitonic transitions. ${ }^{42,43}$ In accordance with the theoretical approach developed in Sec. III, we attribute the peculiarities in the linear absorption spectra and bleaching bands in DT spectra of samples with GaAs, CdSe, and InP nanostructures to linear and nonlinear absorption of either heavy-hole $(h h)$ and light-hole excitons ( $l h$ excitons), i.e., excitons formed with the participation of a hole which is, respectively, heavy and light in the QWR cross-section plane, or $A$ and $B$ excitons formed from the corresponding hole sub-bands of CdSe. Namely, the $h h$ - and $l h$-exciton 


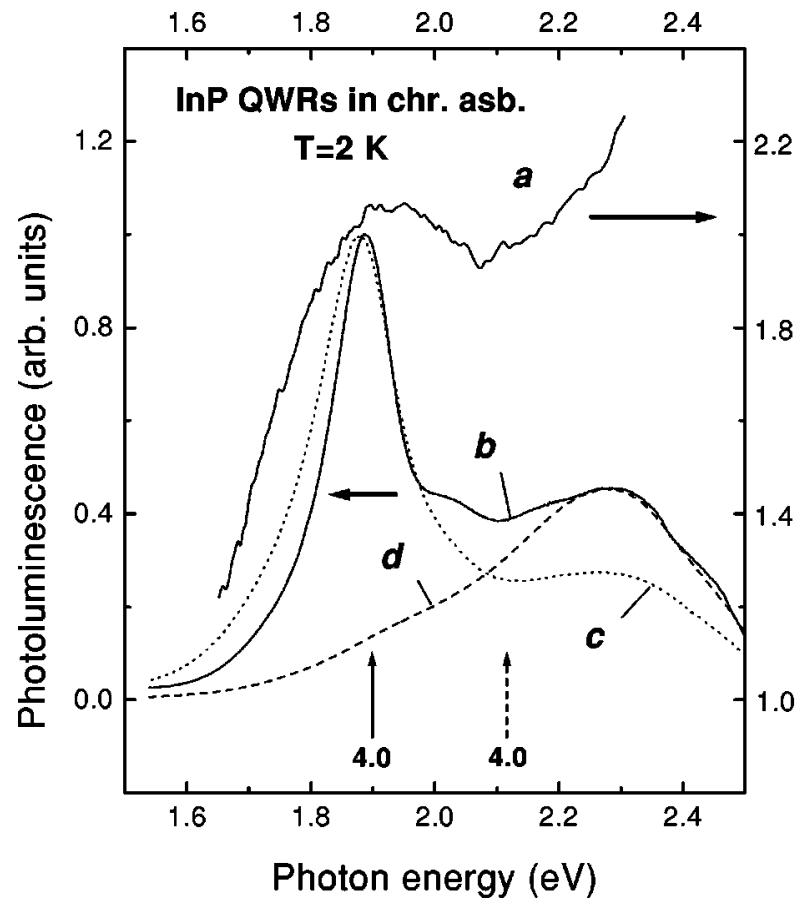

FIG. 6. Absorption spectrum (curve $a$ ), PL spectra (curves $b$ and $c$ ) detected for different areas of a sample with InP in chrysotile-asbestos nanotubes, and PL spectrum of chrysotile asbestos with empty channels (curve $d$ ). The solid and dashed arrows show the calculated exciton transitions for $h h$ and $l h$ excitons, respectively, for InP QWR's $4 \mathrm{~nm}$ in diameter.

transitions in QWR's $4.8 \mathrm{~nm}$ and $6 \mathrm{~nm}$ in diameter describe adequately the features in the spectra of the samples with GaAs. The spectral lines in Fig. 5 are well described by $A$ and $B$-exciton transitions in CdSe QWR's calculated for the wire diameters $d=4 \mathrm{~nm}$ and $4.8 \mathrm{~nm}$, though the transitions in QWR's with $d=6 \mathrm{~nm}$ can also contribute to the absorption. Unfortunately, the spectral broadening, which is very large in the investigated samples does not allow us to separate the contributions of $A$ and $B$ transitions to the spectra. As a result, these pairs of excitonic transitions form wide excitonic absorption bands. Finally, in InP QWR only $h h$ excitons with $d=4 \mathrm{~nm}$ fit the single broad line in the absorption spectrum and PL band centered at $1.892 \mathrm{eV}$ (Fig. 6).

The wide broadening of the exciton transition bands are of both inhomogeneous and structural origin. First, the exciton binding energy and the energies of the dimensional quantization of carriers strongly depend on the wire radius (see Fig. 3 and discussions in Sec. III A). The results of electron microscopy measurements show a notable size dispersion of nanotube diameters, though semiconductor is preferably crystallized in wires with diameters of $4 \mathrm{~nm}, 4.8 \mathrm{~nm}$ and $6 \mathrm{~nm}$, as was already mentioned in Sec. II. Second, in the process of QWR growth there appears structural defects that result in local dispersion of semiconductor parameters, such as energy gap, electron and hole effective masses, etc. The exciton transition energies are found to be very sensitive to variations of these parameters.

A reasonable agreement of the calculated exciton transition energies with that measured in linear absorption $\mathrm{D}, \mathrm{T}$ and PL spectra allow us to estimate the exciton binding energies in GaAs, CdSe, and InP QWR's. In accordance with

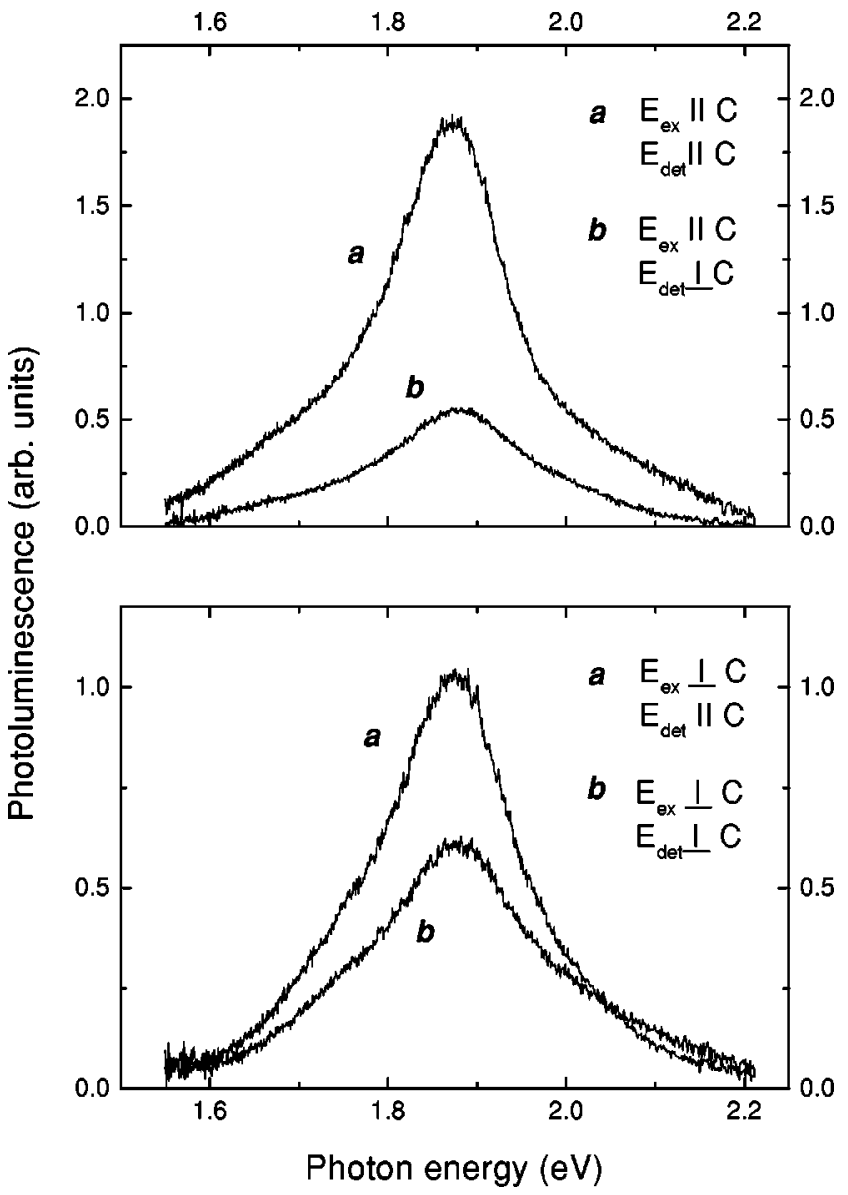

FIG. 7. PL of InP in chrysotile asbestos at $\hbar \omega=1.892 \mathrm{eV}$ for different polarizations of exciting $\left(E_{\mathrm{ex}}\right)$ and probing $\left(E_{\mathrm{det}}\right)$ beams. $C$ is the vector parallel to the QWR axes. The background PL signal of an empty chrysotile asbestos matrix is subtracted.

the calculations of Sec. III, in GaAs QWR's the binding energy of $h h$ exciton $E_{b}=152 \mathrm{meV}$ for $d=4.8 \mathrm{~nm}$ and $E_{b}$ $=127 \mathrm{meV}$ for $d=6.0 \mathrm{~nm}$; in CdSe QWR's $E_{b}=257 \mathrm{meV}$ for $d=4.0 \mathrm{~nm}$ and $E_{b}=224 \mathrm{meV}$ for $d=4.8 \mathrm{~nm}$ ( $A$ exciton); in InP QWR's $E_{b}=192 \mathrm{meV}$ for $d=4.0 \mathrm{~nm}$ ( $h h$ exciton). Thus, we argue that owing to the effect of dielectric enhancement a considerable increase (up to $250 \mathrm{meV}$ ) of the exciton binding energy takes place in the studied samples.

Let us discuss briefly ${ }^{44}$ some nonlinear optical properties of the studied samples. The DT spectra of chrysotile asbestos samples containing CdSe (Fig. 5, curves $b$ and $c$ ) demonstrate both nonlinear absorption of CdSe QWR's and spectral changes near the bulk CdSe absorption edge. The band in the DT spectra positioned at around $1.79 \mathrm{eV}$ corresponds to bulk electron-hole transitions, and its blueshift (compared to the edge of the bulk linear absorption shown by down arrows in Fig. 5) and bleaching under high pumping excitation can be attributed to a renormalization of the semiconductor band gap due to filling of electron and hole bands and effects of Coulomb screening, as well as to the exciton phase-space filling. ${ }^{45,46}$ In linear spectra of all samples we observe an absorption background that monotonously grows with the photon energy. This background is caused by the absorption of relatively large microcrystals on the outer surface of the bundle. With an increase of the optical pumping intensity the changes in the bulk absorption occur only near the funda- 


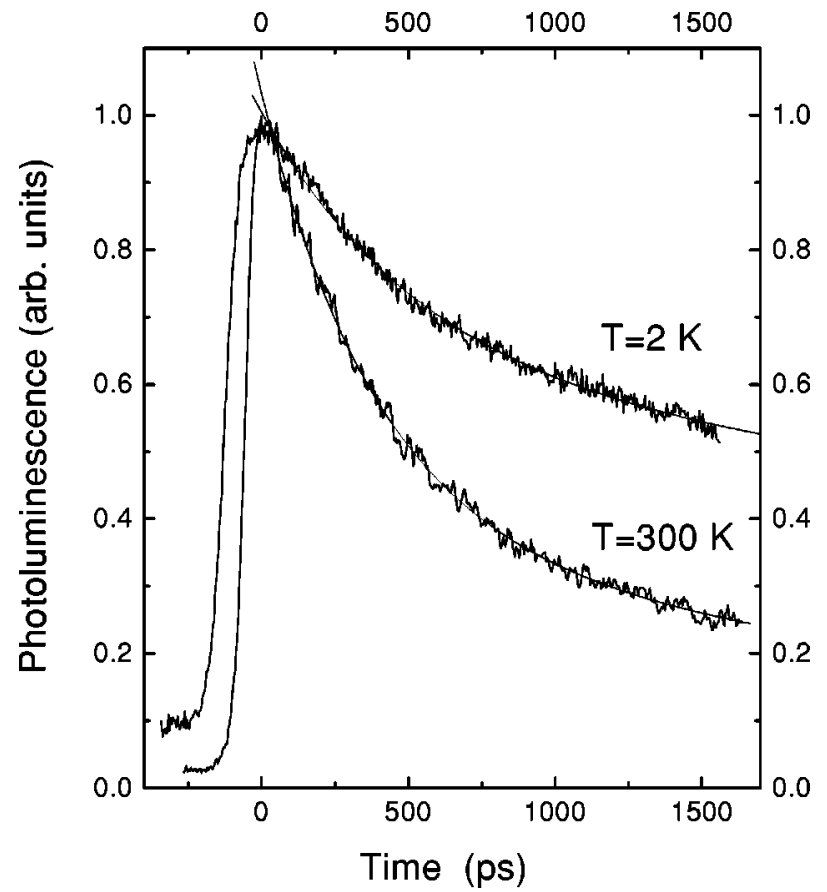

FIG. 8. PL kinetics of InP in chrysotile asbestos at $\hbar \omega=1.892 \mathrm{eV}$. The two-exponent approximation of the time decay behavior is shown by smooth exponential curves.

mental absorption edge after fast relaxation of carriers to the band minimum. This allows us to separate the nonlinear absorption in QWR's from that of the background. The spectral changes at around $2.05 \mathrm{eV}$ in the samples with CdSe as well as three main bleaching bands of DT spectrum of GaAs in chrysotile asbestos, arise due to nonlinear absorption of QWR's. As the optical pumping intensity increases, different nonlinear processes in QWR's coexist and compete with one another, namely, the effects of exciton screening and phasespace filling, the filling of electron and hole energy bands, and renormalization of the band gap in a quasi-onedimensional semiconductor. ${ }^{47,24}$

\section{B. PL anisotropy}

In the investigated samples most of semiconductor nanostructures are elongated QWR-like objects. However, some of them were crystallized as quantum-dotlike structures in a form of short cylinders that can be well approximated by slightly elongated ellipsoids or even spheres. As we have argued in Sec. III D, the absorption and photoluminescence of semiconductor-insulator QWR's possess a significant polarization anisotropy caused by the electric-field redistribution in QWR's, while in spherical quantum dots (QD's) the polarization anisotropy is absent. Thus, the study of the PL anisotropy is a powerful tool to distinguish the features in optical spectra that belong to exciton transitions in QWR's from that of QD's.

We have investigated the anisotropy of polarization of $1.892 \mathrm{eV}$ and $1.796 \mathrm{eV}$ PL bands of InP nanostructures. The PL band positioned near $1.892 \mathrm{eV}$ has a strong polarization anisotropy, while the PL of the $1.796 \mathrm{eV}$ band is almost isotropic. When the polarization vector of the electric field of the exciting light is parallel to the axes of nanotubes, i.e., is along InP QWR's, the intensity of the $1.892 \mathrm{eV}$ band of the
TABLE I. Characteristic time parameters and the corresponding weights calculated in the two-exponent approximation of the PL decay.

\begin{tabular}{lrcccc}
\hline \hline$\hbar \omega, \mathrm{eV}$ & $T, \mathrm{~K}$ & $\tau_{1}, \mathrm{~ns}$ & $\tau_{2}, \mathrm{~ns}$ & $C_{1}$ & $C_{2}$ \\
\hline 1.892 & 2 & 0.55 & 9.8 & 0.40 & 0.60 \\
(QWR's) & 300 & 0.40 & 3.8 & 0.71 & 0.37 \\
1.796 & 2 & 0.48 & 2.3 & 0.42 & 0.58 \\
(QD's) & 300 & 0.19 & 1.2 & 0.81 & 0.25 \\
\hline \hline
\end{tabular}

PL with polarization parallel to QWR's is much larger than that in perpendicular PL polarization (Fig. 7, top): the maximum degree of linear polarization $\sigma=\left(I_{\|}-I_{\perp}\right) /\left(I_{\|}+I_{\perp}\right)$ $=0.55$. In the case of the exciting light polarized perpendicular to the axes of QWR's (Fig. 7, bottom) $I_{\|}$is also greater than $I_{\perp}$, but the maximum degree of linear polarization is smaller: $\sigma=0.25$. So, we attribute the $1.892 \mathrm{eV}$ band to the excitonic PL in QWR's. On the other hand, the fact that the PL polarization anisotropy of the $1.796 \mathrm{eV}$ band is of the order of several percent, strongly supports the transitions occurring in QD's.

Using Eq. (17) we estimate the value of the linear polarization degree for InP QWR's as large as $\sigma=0.76$. This value is found to be somewhat larger than that measured in the experiment. As we do not know the angular dependence of $\left|\mathbf{d}_{c v}\right|$, we assume the dipole matrix element to be isotropic. This is probably far from reality in such highly anisotropic objects as QWR's. There is also a specific depolarization effect that can decrease the calculated value of the PL anisotropy in the samples with InP QWR's. A high polarity of In-P bonds leads to easy rotations of the molecules in the presence of a local electric field and to their orientation along the electrostatic profile. ${ }^{33}$ At the same time, InP molecules on the inner surface of the chrysotile asbestos nanotubes are aligned preferentially in the radial direction due to their interactions with defects on this surface. We believe that the influence of such an induced dipole orientation near the interface may decrease the PL anisotropy.

\section{PL kinetics}

The kinetic properties of QD's and QWR's embedded in the dielectric template are significantly different from that of conventional (semiconductor-semiconductor) nanostructures because of a dominant influence of surrounding material and real interfaces on the electronic system. In such structures the energy relaxation of photoexcited electron-hole pairs and excitons is strongly redistributed between radiative and nonradiative channels of recombination. ${ }^{33,48}$

We have investigated the kinetics of the photoluminescence of InP nanostructures. The time decay of the PL intensity for both $1.892 \mathrm{eV}$ (shown in Fig. 8) and $1.796 \mathrm{eV}$ bands at Helium and room temperatures has fast and slow components at one and the same frequency. For each curve in Fig. 8, the PL intensity of the short-wave band (which we attribute to the excitonic PL in QWR's), as well as that of the long-wave band (QD's), can be well approximated by a twoexponent dependence $I=C_{1} \exp \left(-t / \tau_{1}\right)+C_{2} \exp \left(-t / \tau_{2}\right)$, where the decay parameters $C_{1,2}$ and $\tau_{1,2}$ are presented in Table I. For both bands the relaxation at $2 \mathrm{~K}$ is slower than at 

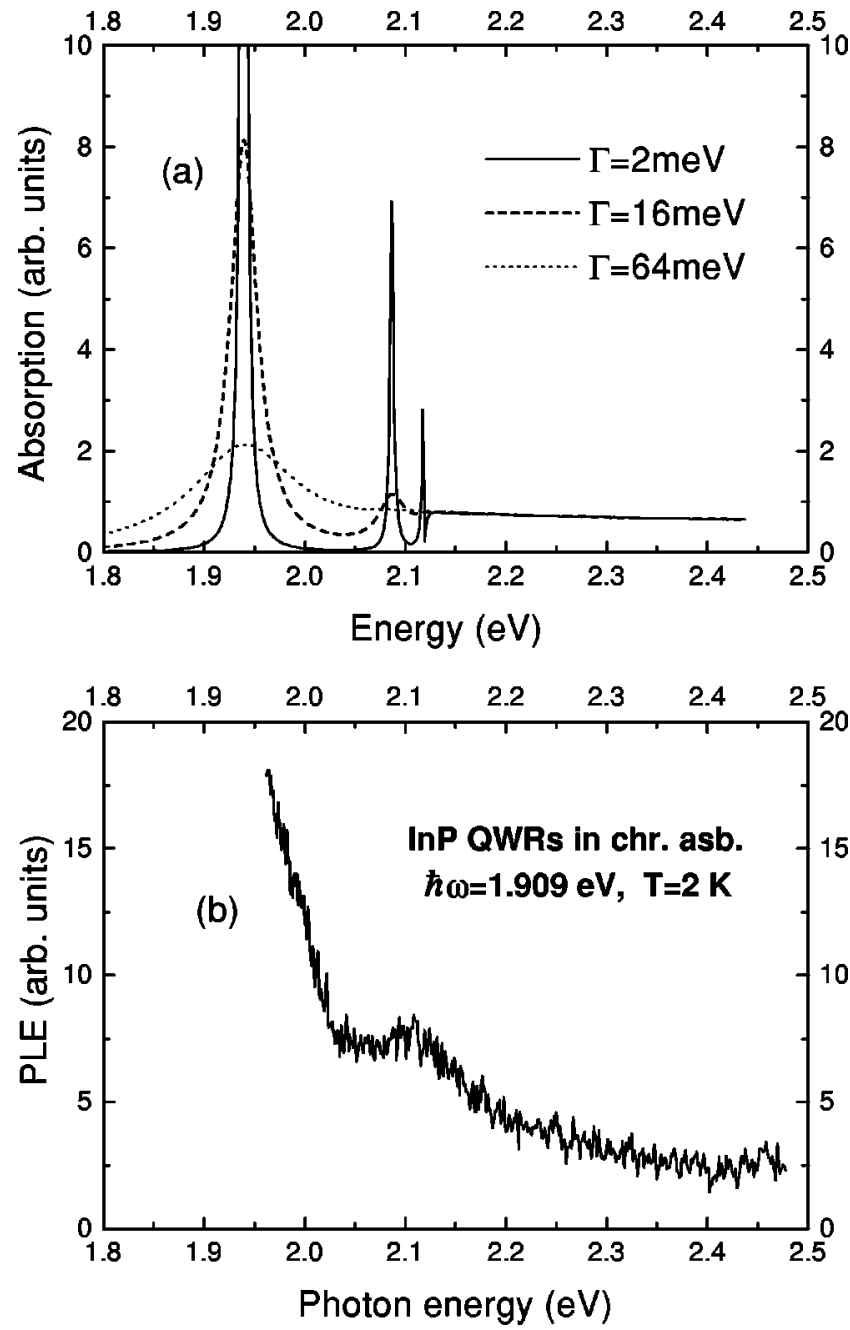

FIG. 9. Absorption spectra of $h h$ exciton in InP QWR $(d=3.8 \mathrm{~nm}$ ) calculated for different values of broadening $\Gamma$ (on the top). PLE spectrum of InP in chrysotile asbestos at $T=2 \mathrm{~K}$ (on the bottom).

$300 \mathrm{~K}$.

The two-component time decay PL in nanostructures has been observed by many experimentalists ${ }^{49-54}$ and treated in terms of concurrent processes of fast recombination inside nanostructures and slow relaxation in the surrounding material and interfaces.

In our experiment the pumping intensity of a Ti-sapphire laser was too low to explain the PL kinetics by any nonlinear process. In InP nanostructures the linear recombination with two different relaxation times at one and the same frequency may be explained by the radiative recombination of carriers (fast component) and by the capture of part of excited carriers on traps on the surface and their subsequent slow recapture (slow component). Both relaxation times are faster for the $1.892 \mathrm{eV}$ band that we attribute to QD's. For QD's the nonradiative recombination may be more efficient because of a stronger influence of surface states.

\section{Photoluminescence excitation spectrum. Direct evaluation of the exciton binding energy}

In our approach we determine the exciton binding energy in semiconductor QWR's in chrysotile asbestos by correla- tion between the exciton transition energies calculated theoretically and that measured from the absorption, DT and PL spectra (Sec. IV A). From the optical spectra we are not able to perform direct measurements of the exciton binding energy since the absorption band edge is not observable in quasi-one-dimensional semiconductors, unlike to three- and two-dimensional structures. This is confirmed by the QWR absorption spectrum calculated in preceding theoretical papers $^{43,55}$ and in this paper, which demonstrates that the exciton transition dominates in semiconductor QWR's and the contribution of the interlevels' transitions to the absorption is very small.

Nevertheless, in semiconductor-insulator QWR's it is possible to evaluate the exciton binding energy from the PLE and absorption spectra. In these systems, due to the dielectric confinement effect, the electron-hole interaction is enhanced by a factor of several tens compared to that in a bulk or even in conventional QWRs. This leads to the possibility that one or several excited states exist in the exciton spectrum and have the oscillator strengths sufficiently large to be observable in the inhomogeneously broadened absorption (or PLE) spectrum.

In Fig. 9(a) two additional peaks corresponding to the excited states are clearly seen in the calculated absorption spectrum of InP QWR's $3.8 \mathrm{~nm}$ in diameter. ${ }^{56}$ The experimental PLE spectrum is shown in Fig. 9(b). A new band in the vicinity of $2.10 \mathrm{eV}$ that is very close to the second absorption peak of the theoretical spectrum calculated with $\Gamma$ $=16 \mathrm{meV}$, has been registered. Assuming that the binding energy of the excited state is small (a theoretical value is less than $50 \mathrm{meV}$ ), we evaluate from this data the ground-state binding energy as large as $200 \mathrm{meV}$.

We would like to note that the energy of the $l h$-exciton transition in InP QWR's calculated for $d=3.8 \mathrm{~nm}$ also falls near the heavy-hole excited state. However, we believe that the energy transfer from light- to heavy-hole exciton state is very small due to rigid selection rules under the strong confinement regime in the investigated QWR's.

\section{CONCLUSIONS}

A theoretical approach to calculations of the exciton parameters in a semiconductor-insulator cylindrical quantum wire of a finite diameter is developed. This approach accounts for the spatial confinement, the self-image effect of renormalization of the wire localizing potentials, and the dielectric enhancement of the electron-hole interaction.

Features of linear absorption, photoluminescence, photoluminescence excitation, time-resolved photoluminescence spectra of GaAs, CdSe, and InP quantum wires imbedded in chrysotile asbestos nanotubes have been detected and interpreted in terms of excitonic transitions. The calculated exciton transition energies and absorption spectra are in good agreement with the experimental results. Owing to the effect of the dielectric enhancement, a considerable increase of the exciton binding energy (up to $260 \mathrm{meV}$ ) has been observed in the studied samples.

A considerable enhancement of the Coulomb interaction leads to more than one exciton bound state appear in optical spectra. Measurements of the energy separation between the ground and excited states in the PLE spectra allow us to 
evaluate the exciton binding energy directly from the experiment.

Combining semiconductor and dielectric materials with different values of dielectric constants, the Coulomb interaction, which is responsible for the exciton binding, may vary over wide limits. This Coulomb interaction engineering opens new possibilities for creation of excitonic devices operating at room temperature.

\section{ACKNOWLEDGMENTS}

We are much indebted to V. V. Poborchii for supplying the samples for our experiments and for critical remarks, to S. G. Romanov, H. M. Yates, and M. E. Pemble for supplying the samples, to N. A. Kiselev and D. N. Zakharov for electronic microscope measurements, to S. G. Tikhodeev for a critical reading of the manuscript, and to $\mathrm{R}$. Zimmermann for useful discussions. The work was supported by the Russian Basic Research Foundation, Russian Ministry of Sciences (program "Nanostructures") and INTAS.

\section{APPENDIX: ELECTROSTATIC POTENTIAL OF A CHARGE IN A SEMICONDUCTOR-INSULATOR CYLINDRICAL WIRE}

To calculate the electrostatic potential $\varphi\left(\boldsymbol{\rho}, \boldsymbol{\rho}_{0}, z-z_{0}\right)$ of a charge $e$ positioned at the point $\mathbf{r}_{0}=\left(\boldsymbol{\rho}_{0}, z_{0}\right)$ we solve the Poisson equation

$$
\Delta \varphi=-\frac{4 \pi e}{\varepsilon_{i}} \delta\left(\mathbf{r}-\mathbf{r}_{0}\right)
$$

with the following boundary conditions

$$
\begin{gathered}
\left.\varphi\right|_{\rho=R-0}=\left.\varphi\right|_{\rho=R+0}, \\
\left.\varepsilon_{\mathrm{S}} \frac{\partial \varphi}{\partial \rho}\right|_{\rho=R-0}=\left.\varepsilon_{\mathrm{I}} \frac{\partial \varphi}{\partial \rho}\right|_{\rho=R+0},
\end{gathered}
$$

where $\varepsilon_{i}=\varepsilon_{\mathrm{S}}$ inside the wire $\rho_{0}=|\boldsymbol{\rho}| \leqslant R$ and $\varepsilon_{i}=\varepsilon_{\mathrm{I}}$ in the outside area $\rho_{0} \geqslant R$ ( $R$ is the wire radius).

Using the cylindrical symmetry of the system and homogeneity along $z$ let us write the solution of Eqs. (A1) and (A2) in the following way

$$
\begin{aligned}
\varphi\left(\mathbf{r}, \mathbf{r}_{0}\right) & \equiv \varphi\left(\rho, \rho_{0}, \theta, z-z_{0}\right) \\
& =\int_{-\infty}^{\infty} d k e^{i k\left(z-z_{0}\right)} \sum_{n=-\infty}^{\infty} e^{i n \theta} \Phi_{n}\left(\rho, \rho_{0}, k\right),
\end{aligned}
$$

where $\theta$ is the angle between $\boldsymbol{\rho}$ and $\boldsymbol{\rho}_{0}$. Then, the Poisson equation in cylindrical coordinates takes the form

$$
\begin{gathered}
\left(-k^{2}+\frac{\partial^{2}}{\partial \rho^{2}}+\frac{1}{\rho} \frac{\partial}{\partial \rho}-\frac{n^{2}}{\rho^{2}}\right) \Phi_{n}\left(\rho, \rho_{0}, k\right) \\
=-\frac{e}{\pi \varepsilon} \frac{1}{\rho} \delta\left(\rho-\rho_{0}\right) .
\end{gathered}
$$

The solution of Eq. (A4) with the boundary conditions Eq. (A2) is a superposition of inhomogeneous and homogeneous parts,

$$
\Phi_{n}=\Phi_{n}^{\mathrm{inhom}}+\Phi_{n}^{\mathrm{hom}}
$$

The inhomogeneous part is nothing else than Fourier transform of Coulomb potential produces by a charge in a homogeneous medium with the dielectric constant $\varepsilon_{i}$ (here $\varepsilon_{i}$ denotes the same as in Eq. (A1),

$$
\Phi_{n}^{\mathrm{inhom}}\left(\rho, \rho_{0}, k\right)=\frac{e}{\pi \varepsilon_{i}} I_{n}\left(k \cdot \min \left\{\rho, \rho_{0}\right\}\right) K_{n}\left(k \cdot \max \left\{\rho, \rho_{0}\right\}\right),
$$

if $\rho, \rho_{0} \leqslant R$ or $\rho, \rho_{0} \geqslant R$, and vanishes in the reminder area: $\Phi_{n}^{\text {inhom }}=0$, if $\rho<R<\rho_{0}$ or $\rho_{0}<R<\rho ; K_{n}(z)$ and $I_{n}(z)$ are the modified Bessel functions. The homogeneous part should be regular at $\rho \rightarrow 0$ and decaying exponentially at $\rho \rightarrow \infty$. Then,

$$
\Phi_{n}^{\text {hom }}\left(\rho, \rho_{0}, k\right)= \begin{cases}C_{n}^{\text {in }}\left(\rho_{0}, k\right) I_{n}(k \rho) & \rho \leqslant R, \\ C_{n}^{\text {out }}\left(\rho_{0}, k\right) K_{n}(k \rho) & \rho>R,\end{cases}
$$

where the constants $C_{n}^{\text {in }}$ and $C_{n}^{\text {out }}$ standing, respectively, in the inside and outside pieces of the potential Eq. (A7) are unambiguously determined from the boundary conditions

$$
\begin{gathered}
\Phi_{n}\left(R-0, \rho_{0}, k\right)=\Phi_{n}\left(R+0, \rho_{0}, k\right), \\
\varepsilon_{\mathrm{S}} \frac{\partial \Phi_{n}}{\partial \rho}\left(R-0, \rho_{0}, k\right)=\varepsilon_{\mathrm{I}} \frac{\partial \Phi_{n}}{\partial \rho}\left(R+0, \rho_{0}, k\right),
\end{gathered}
$$

for any finite $\rho_{0}$. Finally,

$$
\Phi_{n}^{\text {hom }}\left(\rho, \rho_{0}, k\right)=\frac{e}{\pi} \frac{\varepsilon_{\mathrm{S}}-\varepsilon_{\mathrm{I}}}{\varepsilon_{\mathrm{I}} I_{n}(k R) K_{n}^{\prime}(k R)-\varepsilon_{\mathrm{S}} I_{n}^{\prime}(k R) K_{n}(k R)}\left\{\begin{array}{l}
\frac{1}{\varepsilon_{\mathrm{S}}} K_{n}(k R) K_{n}^{\prime}(k R) I_{n}(k \rho) I_{n}\left(k \rho_{0}\right) \quad \text { inside the wire } \\
\frac{1}{\varepsilon_{\mathrm{I}}} I_{n}(k R) I_{n}^{\prime}(k R) K_{n}(k \rho) K_{n}\left(k \rho_{0}\right) \quad \text { outside the wire, }
\end{array}\right.
$$


if $\rho, \rho_{0} \leqslant R$ or $\rho, \rho_{0} \geqslant R$, and

$$
\begin{aligned}
\Phi_{n}^{\text {hom }}\left(\rho, \rho_{0}, k\right)= & \frac{e}{\pi} \frac{I_{n}(k R) K_{n}^{\prime}(k R)-I_{n}^{\prime}(k R) K_{n}(k R)}{\varepsilon_{\mathrm{I}} I_{n}(k R) K_{n}^{\prime}(k R)-\varepsilon_{\mathrm{S}} I_{n}^{\prime}(k R) K_{n}(k R)} \\
& \times I_{n}\left(k \cdot \min \left\{\rho, \rho_{0}\right\}\right) K_{n}\left(k \cdot \max \left\{\rho, \rho_{0}\right\}\right)
\end{aligned}
$$

in the rest region. $K_{n}^{\prime}$ and $I_{n}^{\prime}$ in Eqs. (A9) and (A10) are the first derivatives of the modified Bessel functions.
At small $k$, i.e., when $k R, k \rho, k \rho_{0} \ll 1$, the zeromomentum Fourier transform takes the form

$$
\begin{aligned}
\Phi_{0}\left(\rho, \rho_{0}, k\right)= & -\frac{e}{\varepsilon_{\mathrm{I}}}\left(\ln \frac{|k| R}{2}+C\right)-\frac{e}{\pi \varepsilon_{i}} \ln \frac{\max \left\{\rho, \rho_{0}\right\}}{R} \\
& +O\left[\frac{\varepsilon_{\mathrm{S}}}{\varepsilon_{\mathrm{I}}}(k R)^{2} \ln (|k| R)\right],
\end{aligned}
$$

where $\varepsilon_{i}=\varepsilon_{\mathrm{S}}$ if $\rho, \rho_{0} \leqslant R$ and $\varepsilon_{i}=\varepsilon_{\mathrm{I}}$ in the rest region, and $C$ is Euler's constant.
${ }^{1}$ E. Kapon, Proc. IEEE 80, 398 (1992).

${ }^{2}$ W. Weigscheider, L. N. Pfeiffer, M. M. Dignam, A. Pinczuk, K. W. West, S. L. McCall, and R. Hull, Phys. Rev. Lett. 71, 4071 (1993)

${ }^{3}$ T. Someya, H. Akiyama, and H. Sakaki, Phys. Rev. Lett. 76, 2965 (1996).

${ }^{4}$ H. Weman, M. Potemski, M. E. Lazzouni, M. S. Miller, and J. L. Merz, Phys. Rev. B 53, 6959 (1996).

${ }^{5}$ S. Glutsch, F. Bechstedt, W. Wegscheider, and G. Schedelbeck, Phys. Rev. B 56, 4108 (1997).

${ }^{6}$ L. V. Keldysh, Phys. Status Solidi A 164, 3 (1997).

${ }^{7}$ D. Brinkman and G. Fishman, Phys. Status Solidi A 164, 401 (1997).

${ }^{8}$ S. Glutsch, F. Bechstedt, W. Wegscheider, and G. Schedelbeck, Phys. Status Solidi A 164, 405 (1997).

${ }^{9}$ V. S. Babichenko, L. V. Keldysh, and A. P. Silin, Fiz. Tverd. Tela (Leningrad) 22, 1238 (1980) [Sov. Phys. Solid State 22, 723 (1980)].

${ }^{10}$ E. A. Muljarov and S. G. Tikhodeev, Zh. Éksp. Teor. Fiz. 111, 274 (1997) [JETP 84, 151 (1997)].

${ }^{11}$ N. S. Rytova, Dokl. Akad. Nauk SSSR 163, 1118 (1965) [Sov. Phys. Dokl. 10, 754 (1966)].

${ }^{12}$ A. V. Chaplik and M. V. Entin, Zh. Éksp. Teor. Fiz. 61, 2496 (1971) [JETP 61, 2469 (1971)].

${ }^{13}$ L. V. Keldysh, Pis'ma Zh. Éksp. Teor. Fiz. 29, 716 (1979) [JETP Lett. 29, 658 (1979)].

${ }^{14}$ E. Hanamura, N. Nagaosa, M. Kumagai, and T. Takagahara, Mater. Sci. Eng., B 1, 255 (1988).

${ }^{15}$ M. Kumagai and T. Takagahara, Phys. Rev. B 40, 12359 (1989).

${ }^{16}$ D. B. Tran Thoai, R. Zimmermann, M. Grundmann, and D. Bimberg, Phys. Rev. B 42, 5906 (1990).

${ }^{17}$ L. Wendler and B. Hartwig, J. Phys.: Condens. Matter 3, 9907 (1991).

${ }^{18}$ X. Zhang, Y. Li, Z. Kong, and Ch. Wei, Phys. Rev. B 49, 10432 (1994).

${ }^{19}$ J. Cen, R. Chen, and K. K. Bajaj, Phys. Rev. B 50, 10947 (1994).

${ }^{20}$ R. R. Guseinov, Phys. Status Solidi B 125, 237 (1984).

${ }^{21}$ E. A. Muljarov, S. G. Tikhodeev, N. A. Gippius, and T. Ishihara, Phys. Rev. B 51, 14370 (1995)

${ }^{22}$ T. Takagahara, Phys. Rev. B 47, 4569 (1993).

23 T. Ishihara, in Optical Properties of Low-Dimensional Materials, edited by T. Ogawa and Y. Kanemitsu (World Scientific, Singapore, 1995).

${ }^{24}$ V. S. Dneprovskii, E. A. Zhukov, E. A. Muljarov, and S. G.
Tikhodeev, Zh. Éksp. Teor. Fiz. 114, 700 (1998) [JETP 87, 382 (1998)].

${ }^{25}$ P. Ils, Ch. Greus, A. Forchel, V. D. Kulakovskii, N. A. Gippius, and S. G. Tikhodeev, Phys. Rev. B 51, 4272 (1995).

${ }^{26}$ L. V. Kulik, V. D. Kulakovskii, M. Bayer, A. Forchel, N. A. Gippius, and S. G. Tikhodeev, Phys. Rev. B 54, 2335 (1996).

${ }^{27}$ N. A. Gippius, A. L. Yablonskii, A. B. Dzyubenko, S. G. Tikhodeev, L. V. Kulik, and V. D. Kulakovskii, J. Appl. Phys. 83, 5410 (1998).

${ }^{28}$ G. Goldoni, F. Rossi, and E. Molinari, Phys. Rev. Lett. 80, 4995 (1998).

29 V. N. Bogomolov, Usp. Fiz. Nauk 124, 171 (1978) [Sov. Phys. Usp 21, 77 (1978)].

${ }^{30}$ V. V. Poborchii, M. S. Ivanova, and I. A. Salamatina, Superlattices Microstruct. 16, 133 (1994).

${ }^{31}$ V. V. Poborchii, V. I. Al'perovich, Y. Nozue, N. Ohnishi, A. Kasuya, and O. Terasaki, J. Phys.: Condens. Matter 9, 5687 (1997).

${ }^{32}$ H. M. Yates, M. E. Pemble, N. P. Johnson, S. G. Romanov, and C. M. Sotomayor-Torres, J. Cryst. Growth 170, 611 (1997).

${ }^{33}$ S. G. Romanov, C. M. Sotomayor-Torres, H. M. Yates, M. E. Pemble, V. Butko, and V. Tretijakov, J. Appl. Phys. 82, 380 (1997).

${ }^{34}$ P. Christol, P. Lefebre, and H. Mathieu, J. Appl. Phys. 74, 5626 (1993).

${ }^{35}$ J. M. Luttinger, Phys. Rev. 102, 1030 (1956).

${ }^{36}$ Landolt-Börnstein, New Series, Group III (Springer-Verlag, Berlin, 1982), Vol. 17.

37 The using of the high frequency dielectric constant is justified by the fact that in the investigated samples the exciton binding energies are much larger than the LO phonon frequencies in all materials.

${ }^{38}$ V. V. Poborchii, Jpn. J. Appl. Phys. 34, Suppl. 34-1, 228 (1995).

${ }^{39}$ D. Kovalev, M. Ben Chorin, J. Diener, F. Koch, Al. L. Efros, M. Rosen, N. A. Gippius, and S. G. Tikhodeev, Appl. Phys. Lett. 67, 1585 (1995).

${ }^{40}$ L. D. Landau and E. M. Lifshitz, Electrodynamics of Continuous Media (Pergamon, Oxford, 1960).

${ }^{41}$ R. Zimmermann, Phys. Status Solidi B 135, 681 (1986).

${ }^{42}$ T. Ogawa and T. Takagahara, Phys. Rev. B 43, 14325 (1991); 44, 8138 (1991).

${ }^{43}$ S. Glutsch and D. S. Chemla, Phys. Rev. B 53, 15902 (1996).

${ }^{44}$ For more details see in Ref. 24.

${ }^{45}$ V. D. Egorov, H. X. Nguyen, R. Zimmermann, V. S. 
Dneprovskii, M. Kaschke, and D. S. Khechinashvili, Phys. Status Solidi B 159, 403 (1990).

${ }^{46}$ H. Haug and S. W. Koch, Quantum Theory and Electronic Properties of Semiconductors (World Scientific, Singapore, 1990).

${ }^{47}$ S. Benner and H. Haug, Europhys. Lett. 16, 579 (1991).

${ }^{48}$ E. A. Zhukov, Y. Masumoto, E. A. Muljarov, and S. G. Romanov, Solid State Commun. 112, 575 (1999).

${ }^{49}$ M. G. Bawendi, P. J. Carroll, W. L. Wilson, and L. E. Brus, J. Chem. Phys. 96, 946 (1992).

${ }^{50}$ W. Yang, R. R. Lowe-Webb, H. Lee, and P. C. Sercel, Phys. Rev. B 56, 13314 (1997).

${ }^{51}$ H. Yu, S. Lycett, C. Roberts, and R. Murray, Appl. Phys. Lett. 69,
4087 (1996).

${ }^{52}$ S. Raymond, S. Fafard, P.J. Poole, A. Wojs, P. Hawrylak, and S. Charbonneau, Phys. Rev. B 54, 11548 (1996).

${ }^{53}$ G. Wang, S. Fafard, D. Leonard, J. E. Bowers, J. L. Merz, and P. M. Petroff, Appl. Phys. Lett. 64, 2815 (1994).

${ }^{54}$ T. Okuno, H. -W. Ren, M. Sugisaki, K. Nishi, S. Sugou, and Y. Masumoto, Phys. Rev. B 57, 1386 (1998).

${ }^{55}$ R. Zimmermann, Jpn. J. Appl. Phys. 34, Suppl. 34-1, 228 (1995).

56 In the PLE experiment the frequency of the monochomator $(1.909 \mathrm{eV})$ was adjusted on the high-energy tail of the PL band, thus the signal of the exciton PL was registered only from QWR's with the diameter less than $4 \mathrm{~nm}$. 